\title{
THE TREND OF CARDIOLOGY
}

\author{
By William Evans, M.D., D.Sc., F.R.C.P. \\ Physician to the Cardiac Department of the London Hospital and to the National Heart Hospital, Consulting Cardiologist \\ to the Royal Naz'y
}

The study of cardiovascular disease has increased both in its scope and pace during the last decade. Included amongst the circumstances which have contributed to these changes are the recruitment to the specialty of younger workers on leaving the Fighting Services, the provision of greater facilities for research work, and the addition of newer instruments and equipment in the investigation of the several problems which await solution, while the application of surgery to the treatment of certain cardiovascular disorders has given a sharp incentive to the impatient as well as to the thoughtful therapist.

The pages that follow will show how heavy has been the impact of such influences on orthodox clinical cardiology, and they should help opinion on the need to forego old views, or to form new ones, about cardiovascular disease. Here I only intend to touch on the changes which modern methods of investigation and therapy may bring about and to try and weigh their merits; it will be convenient to consider them under four headings.

\section{Cardiac Pain}

The immediate years ahead should bring help in the readier diagnosis of cardiac pain having its source in diseased coronary arteries. A careful history of the site and distribution of the pain and its relation to effort, cold, meals and to resting, useful as it might be, cannot decide by itself the cause of the pain nor the presence or absence of coronary artery disease. Attention to the physical signs, however, may provide the diagnosis. Thus a raised blood pressure which induces or increases coronary atheroma, or a low blood pressure from cardiac infarction, will sometimes give a clue. The presence of aortic incompetence will suggest obstruction of the coronary orifices by aortitis. Pericardial friction sound, telling of cardiac infarction is too infrequent to be of much value. A physical sign of the greatest importance, both on account of the ease with which it is elicited and the surety of its implication, is a triple heart rhythm from addition of the third heart sound at the lower end of the sternum. In a patient with chest pain the presence of this added sound points unerringly to the diagnosis of coronary artery disease. A further contribution to the recognition of cardiac pain comes from finding some degree of cardiac enlargement, usually detected only on radiological examination, and this may sometimes happen alongside pulmonary congestion which in turn might be a lone sign.

It is unwise to dogmatize on the cause of chest pain, in terms of coronary artery disease, after attending only to the clinical history and to a physical examination which has been barren of signs, without recourse to electrocardiography. In the face of our eagerness to follow our clinical judgment and to be independent of laboratory tests, we should admit that such an attitude in this circumstance invites disaster, and that a patient with pain behind the breastbone is a neglected patient if the electrocardiogram has not been examined as well. Chest leads in addition to limb leads need to be taken and in this directions cardiology has travelled far within the last feve years. It is unlikely that all which has been saifo recently about chest lead electrocardiography couched in terms of electrodynamics, need be assimilated by the clinician seeking a diagnosis of his patient's pain; rather must he apply the test purposefully in the discovery of the patient's complaint expressed in terms of structural heart disease and to bring it within the compass of recognized therapeutic measures.

In the treatment of cardiac pain the influence of anticoagulant medicines on the course of the illness will have to be decided. In the meantime this might be said before this form of therapy is too readily accepted. First, it is a good precept in dealing with any illness to prescribe a remedy which can do no harm and may do good, but to withhold any drug whose good effects are as yet unproven and whose harmful action has often been exhibited. Secondly, when the safe therapeutic control of a drug is notably difficult, and such is the case with heparin and dicoumarol, we should exercise special care in testing its undoubted efficiency in the treatment of any condition. It is my view that anticoagulants are unlikely to prove a valuable agent in the management of cardiac pain.

\section{Congenital Heart Disease}

The adventure of surgery in this section of 
cardiology has changed the complexion of things, and this has particularly demanded of the physician a more precise diagnosis of the congenital anomaly which is present. Apart from a sharper appreciation of clinical signs and the findings of phonocardiography, electrocardiography and cardioscopy, two newer methods of investigation have come to the front, namely, cardiac catheterization and angiocardiography. Deductions from these tests are not as yet infallible; intracardiac pressure estimated by the former method can hardly be accurate because of the great length and the small bore of the catheter, while the second method is not free from danger. Improvement in the technique connected with both methods is certain to take place, but it is likely that they can best serve cardiology by apportioning to clinical signs a newer merning in diagnosis: and that they should then fall out as methods for the routine investigation of cardiovascular disorders.

The indications for the surgical treatment of congenital cardiovascular defects remain haphazard, and the immediate years ahead will define these more clearly. It is becoming common practice to ligate the patent ductus arteriosus whenever it is met with in children. Is this necessary or even advisable? It may be so, but a study of the natural history of this anomaly may show that it sometimes closes spontaneously with the years. Certainly the incidence of the condition in children is very much higher than in young and older adults. Again, when surgeons gain wider experience in the radical treatment of coarctation of the aorta it will be necessary to speak definitely on the need for this venturesome operation. Two things in the meantime discourage enthusiasm for this procedure, namely, the longevity of some patients in the absence of any active interference with the lesion, and the uncertain behaviour of the ringed scar in the aorta with ageing of the patient and especially in those where hypertension may develop. The relief of cyanosis in Fallot's syndrome by creating a fistula between the aorta or subclavian artery and the pulmonary artery is a noteworthy project undertaken with a good deal of risk. Its advisability may well have to be determined on aesthetic grounds; is it best to do nothing and to hope for a moderate span of life denuded of natural physical activities, or is it better to face the immediate hazards of the operation and the more distant duress imposed on the heart by the creation of the artificial fistula in order to gain alleviation of the cyanosis and improved locomotion for a time? The decision has to be made often, and a few more years' experience will help us to make it with less hesitation than has now to be shown.

\section{Appraisal of Heart Murmurs}

The interpretation of murmurs in terms of $\frac{T}{8}$ structural heart disease has been helped in the past by findings on radiological examination or at necropsy. More recently it has been improved by a more systematized use of the phonocardiograph. So far the instrument is not standardized, but soon it should develop into a dependable unit when it will unerringly show the position of sounds and murmurs in successive cardiac cycles. When the instrument matures the test will give us help not far short of the electrocardiogram. Already it has placed in our hands a sure means of telling innocent murmurs.

\section{The Diagnosis and Treatment of Hyper- tension}

Reliance has been placed in the past on the manometric reading as a sign, and often the only sign, of systemic hypertension; insurance companies through the years have framed their policy of acceptance with loaded premium, or rejection, of applicants for life insurance wholly on this variable physiological test. To ignore the signs of cardiovascular hypertrophy which are inseparable from a diagnosis of hypertension is blameworthy: the practice should not be allowed to go on much longer, and there is need of a definition of hyperê tension which will ensure the admission of in? disputable evidence of this condition whenever its. presence has to be tested.

Before long we shall be set the task of deciding unequivocally on the benefit that dorso-lumbar sympathectomy can bring to a patient with hypertension. One thing the operation can do is to improve the changes which may have taken place in the retina both in simple and papilloedemic hypertension. A hypotensive effect is not always brought about by the operation. In papilloedemic or malignant hypertension it is doubtful whether the advocation of surgery in its treatment would be so strong if it had not been for the knowledge of the forlorn outlook in such cases following any other form of treatment. In simple hypertension it is difficult to match the improvement which might be the outcome of a satisfactory operation against the natural longevity which is the fate of so many patients with this relatively benign form of hypertension, and in the absence of an operation which by itself can produce distressing symptoms.

All the problems engaging the cardiologist have not been mentioned, but some of the more urgent ones have been outlined. A solution to most of them seems imminent, but doubtless others will arise anew to take their place and to tax, and in turn yield to, the ingenuity of the investigator in this field of medicine. 